\title{
Feasibility of Carbon Ion Radiotherapy in the Treatment of Gynecological Melanoma
}

\author{
AMELIA BARCELLINI ${ }^{1}$, VIVIANA VITOLO ${ }^{1}$, ANGELICA FACOETTI ${ }^{1}$, PIERO FOSSATI $^{1}$, \\ LORENZO PREDA $^{1,2}$, MARIA ROSARIA FIORE ${ }^{1}$, BARBARA VISCHIONI $^{1}$, ALBERTO IANNALFI $^{1}$, \\ MARIA BONORA ${ }^{1}$, SARA RONCHI ${ }^{1}$, EMMA D' IPPOLITO ${ }^{1}$, RACHELE PETRUCCI $^{1}$, \\ GISELA VISELNER $^{1}$, MARIO CIOCCA ${ }^{1}$, FRANCESCA VALVO $^{1}$ and ROBERTO ORECCHIA ${ }^{1,3}$ \\ ${ }^{1}$ National Center of Oncological Hadrontherapy, Pavia, Italy; \\ ${ }^{2}$ Department of Clinical-Surgical, Diagnostic and Pediatric Sciences, University of Pavia, Pavia, Italy; \\ ${ }^{3}$ European Institute of Oncology, Milan, Italy
}

\begin{abstract}
Background: Malignant melanoma of the lower genital tract is a rare disease known to have a poor prognosis. Because of the high rate of distant metastasis and unsatisfactory survival benefit, a more conservative treatment approach, instead of extensive surgery, may be warranted. Gynecological melanoma is a radioresistant tumor, an ideal disease to test the biological efficacy of carbon ion radiotherapy (CIRT). Aim: To report our preliminary experience with CIRT in the treatment of gynecological melanoma at the National Center of Oncological Hadrontherapy (CNAO). Patients and Methods: Between January 2016 and February 2017, four patients were admitted for CIRT at CNAO. A case of cervical melanoma was treated with palliative aim because of large volume macroscopic disease, while three cases of vaginal melanoma were irradiated with a total dose of 68.8 Gy (relative biological effectiveness) in 16 fractions delivered over 4 weeks (4 days a week). Results: The age of women ranged between 49 and 72 (median=60.5 years) years. Treatment was well tolerated in all patients and all women completed the scheduled treatment course. During CIRT, toxicity was mild. For patients with vaginal disease, local control was 10.23 and 12.6 months, while that for cervical malignant melanoma was 7.3 months. All patients experienced systemic progression, with median distant metastasis-free survival of 11.7 months. The median overall
\end{abstract}

This article is freely accessible online.

Correspondence to: Angelica Facoetti, Fondazione CNAO, Strada Campeggi 53, Pavia, Italy. Tel: +39 0382078501, Fax: +39 0382078903, e-mail: Facoetti@cnao.it

Key Words: Gynecological melanoma, lower genital tract, carbon ion radiotherapy. survival for the whole patient group was 11.41 months. Conclusion: In our first experiences, CIRT appears to be a safe non-invasive option for malignant melanoma of the lower genital tract, but more data and longer follow-up are necessary in order to evaluate the effectiveness and late effects.

Melanomas of the female lower genital tract are a rare and aggressive variety of malignant mucosal melanoma (MMM). Of all gynecological MMM, 95\% arise in the vulva, $3 \%$ originate within the vagina, and it is much rarer in the cervix. Analysis of literature reported an annual ageadjusted incidence ranging from 0.87 to 1.90 per $1,000,000$, greater in non-Hispanic Whites and lower in Black women (1).

The clinical outcome is very poor due to frequent distant metastases with an estimated 5-year survival of 10\%, 13$32.3 \%$ and $37-50 \%$ for cervical, vaginal and vulvar MMM respectively (1).

The main prognostic factors are tumor size, tumor stage, nodal status and, for vulvar MMM the Breslow's thickness $(2-7)$

Due to their rarity and poor overall survival, there is no evidence-based management for gynecological MMM and the treatment strategy, as recommended by the Gynecologic Cancer InterGroup (8), is extrapolated from the data for cutaneous melanoma, even though medical therapy should consider the distinct genetic profile and mutational burden of melanoma at this particular site $(9,10)$.

Surgery is the standard treatment when feasible and the procedure may range from a radical to a more conservative approach. Due to the high rate of distant metastasis and unsatisfactory survival benefit, more conservative treatment approaches, instead of extensive surgery, may be warranted. Melanomas are resistant to conventional radiotherapy but, due to their biological and physical advantages, therapy with 
charged particles may represent an interesting treatment option. In the Japanese clinical trial of radical carbon ion radiotherapy (CIRT) for gynecological melanoma, the 3-year local control (LC) rate was promising (49.9\%) with mild morbidity (11).

We report our preliminary experience with CIRT for treatment of gynecological melanoma at the National Center of Oncological Hadrontherapy (CNAO).

\section{Patients and Methods}

Between January 2016 and February 2017, three patients with vaginal MMM and one with cervical MMM were admitted for CIRT at CNAO. All patients underwent staging with a total body computed tomographic (CT) scan and a pelvic magnetic resonance imaging (MRI). All patients were treated with synchrotron-based scanning carbon ion beams (pencil beam scanning and spill-by-spill active energy variation). Details on CNAO particle delivery system have been previously reported $(12,13)$.

Each patient was immobilized in the supine position with a custom body thermoplastic mask. A set of CT images of $2 \mathrm{~mm}$ thickness was acquired for treatment planning. Contrast-enhanced MRI was performed for precise gross tumor volume (GTV) identification. MRI scans were performed in the treatment position and with immobilization devices, using dedicated protocols. MRI scans were rigidly registered to planning CT.

Prescription doses and fractionation scheme were based on the National Institute of Radiological Sciences (Chiba, Japan) experience translated according to the dose conversion approach described in Fossati et al. (14) and Molinelli et al. (15).

The CE-marked Syngo RT Planning treatment planning system (Siemens AG Healthcare, Erlangen, Germany), version C13, was used for plan optimization and calculation of relative biological effectiveness (RBE)-weighted dose distributions according to the Local Effect Model (LEM) version I, with the following parameters: $\alpha_{\gamma}=0.1 \mathrm{~Gy}^{-1}, \beta_{\gamma}=0.05 \mathrm{~Gy}^{-2}, \mathrm{Dt}=30 \mathrm{~Gy}$, nuclear radius $=5 \mu \mathrm{m}$ (16).

Intensity-modulated particle therapy was employed as plan optimization strategy $(17,18)$. Once approved, each plan was checked by a medical physicist according to our institutional protocol for patient-specific pre-treatment dosimetric verification (19). Patient set-up was verified at each treatment session by means of two independent custom verification systems, namely an infrared optical tracking system and a stereoscopic $\mathrm{x}$-ray verification device (20). Six-degrees of freedom set-up correction vector was calculated and remotely applied to the treatment table.

As regards vaginal MMM, the clinical target volume (CTV) 1 was defined as the inguinal lymph nodes and the small pelvis (internal iliac, external iliac, obturator lymph nodes) including the GTV with a minimum margin of $5 \mathrm{~mm}$, which was irradiated with 38.7 Gy RBE in nine fractions. CTV2, was equal to the GTV with a minimum margin of $5 \mathrm{~mm}$ and was irradiated up to a total dose of $68.8 \mathrm{~Gy} \mathrm{RBE}$ in 16 fractions (Figure 1). Limiting dose for organ at risks was 60 Gy RBE for the rectum.

The patient with cervical MMM was treated with a palliative dose of 24 Gy RBE in three fractions and, because of the large volume of macroscopic disease, the CTV was defined as the uterine cervix and corpus.

Toxicity was scored according to Common Terminology Criteria for Adverse Events version 4.0 (21). Time to event data were calculated from the end of CIRT.

\section{Results}

Patient and tumor characteristics are described in Table I. The median age of women was 60.5 (range=49-72) years and the Karnofsky Performance Status was $>90$. All patients were wild-type for V-Raf murine sarcoma viral oncogene homolog B1 $(B R A F) /$ neuroblastoma RAS viral oncogene homolog $(N R A S)$, and tyrosine kinase $\mathrm{C}(c-K I T)$ mutation was identified in one vaginal MMM. No neoadjuvant or concomitant chemotherapy was administered.

Patients with vaginal MMM had no nodal metastases and the maximum tumor extension observed by imaging/ gynecological bimanual evaluation was $37 \mathrm{~mm}, 12 \mathrm{~mm}$ and $5 \mathrm{~mm}$, respectively, corresponding to GTV of $28.01 \mathrm{cc}, 25.59$ $\mathrm{cc}$ and $1.2 \mathrm{cc}$.

The cervical MMM invaded the bladder and rectum, showed an extension of $80 \times 90 \times 100 \mathrm{~mm}$ and a GTV of $380.96 \mathrm{cc}$.

One patient with vaginal MMM underwent CIRT for recurrent disease after surgery (after a disease-free survival of 11 months). Treatment was well tolerated and no interruption was necessary.

As regards toxicity, during and at the end of CIRT, one patient experienced grade 3 erythema and two patients grade 1 vaginitis, one woman developed grade 2 vaginitis 3 months after the end of CIRT; in evaluable patients, no late toxicity more than grade 2 was experienced.

Only one patient affected by vaginal MMM, who did not experience systemic progression, remains alive. She underwent colpectomy and hysterectomy 5.6 months after the end of CIRT for suspected local progression of disease. The pathologist described residual disease (Breslow's thickness: $0.93 \mathrm{~mm}$ ). For the patients with vaginal MMM, LC was 10.23 and 12.6 months, and that for cervical MMM was 7.3 months. The median distant metastasis-free survival was 11.7 months and the median overall survival was 11.41 months (range=9.76-18.77 months). The patient with cervical MMM and the two patients who experienced systemic progression of vaginal MMM received chemotherapy.

\section{Discussion}

No standard treatment for gynecological MMM has been established in the literature. Surgical excision either conservative or radical remains the mainstay of treatment, even in cases of radical excision, prognosis of female genital MMM remains poor. For these reasons, a conservative local approach may be justified. Typically, melanoma is considered resistant to photon radiotherapy, but CIRT overcomes this biological resistance, reducing the oxygen enhancement ratio and diminishing both the capacity for sublethal repair and cell cycle-dependent radiosensitivity (22).

The Japanese experience in CIRT for gynecological MMM appears encouraging. In fact, after a median follow-up of 17 
Table I. Patient, tumor and treatment characteristics.

\begin{tabular}{lc}
\hline & Value \\
\hline Age, years & \\
$\quad$ Median (range) & $60.5(49-72)$ \\
Tumor site & \\
$\quad$ Vagina & 3 \\
Cervix uteri & 1 \\
Molecular features & \\
$B R A F$ mutation & 0 \\
KRAS mutation & 0 \\
$c K I T$ mutation & 1 (Vaginal melanoma) \\
Previous treatment & 1 (Vaginal melanoma) \\
Surgery & 380.96 \\
GTV volume, cc & 18.01 (1.2-28.01) \\
Cervical MMM & \\
Vaginal MMM mean (range) & 24 Gy RBE in 3 fractions \\
Total dose & \\
Cervical MMM & \\
Vaginal MMM & \\
Pelvic space & 38.7 Gy RBE in 9 fractions \\
GTV boost & 30.1 Gy RBE in 7 fractions \\
\hline
\end{tabular}

$B R A F$ : V-Raf murine sarcoma viral oncogene homolog B1; NRAS: neuroblastoma RAS viral oncogene homolog; $c$-KIT: tyrosine kinase C; MMM: malignant mucosal melanoma; GTV: gross tumor volume; RBE: relative biological effectiveness. aSee Figure 1.

months, the 3-year LC and regional control rates of the 23 patients were $49.9 \%$ and $76.1 \%$ respectively and, as regards the initial tumor response, the maximum effect within 6 months was complete response in six and partial response in 17. In terms of recurrence, six patients experienced local recurrence (three in CTV and three in marginal regions), five had recurrence in regional lymph nodes and 11 in distant organs. In these patients, the acute and late toxicity was mild, with only one case of acute and late reactions in a woman who received dacarbazine with nimustine hydrochloride and vincristine sulfate and concurrent Feron. This patient had no local or regional recurrence, but had lung metastases 15 months after the completion of CIRT. The analysis of response by site showed a statistical difference in LC between the uterine cervix ( $0 \%$ at 1.3 years) and vagina (55.8\% at 3 years) $(p=0.009)$, and the vulva $(100 \%$ at 2.5 years $)(p=0.027)(11)$.

Based on these promising Japanese results in the treatment of gynecological MMM and in light of the well-known biological and therapeutic advantages of carbon ions, we were prompted to use CIRT for gynecological MMM. Despite the few patients treated so far, treatment appears safe and feasible: it was well tolerated and toxicity did not affect the quality of life of patients assessed during clinical follow-up. From May 2018, another four women affected by gynecological MMM were admitted for CIRT, data on their follow-up is still ongoing.

For patients affected by gynecological MMM, due to poor clinical outcome, CIRT should be a promising alternative to

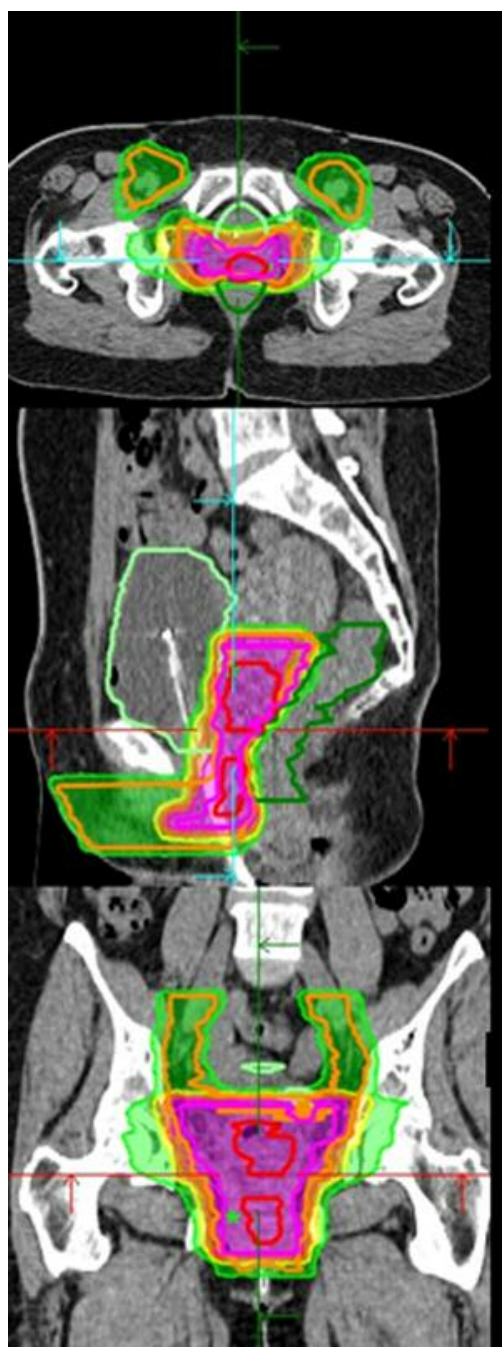

Figure 1. A case of vaginal melanoma treated with carbon ion radiotherapy. The clinical target volume (CTV) 1 (orange line) was irradiated with up to a total dose of 38.7 Gy relative biological effectiveness (RBE) in nine fractions, followed by a boost of up to a total dose of 68.8 Gy RBE to CTV2 (fuchsia line).

other aggressive procedures and patients should be encouraged to undergo to such a clinical pathway. The use of immunecheckpoint inhibitors (nivolumab and ipilimumab) and targeted agents such as c-KIT inhibitors (imatinib), BRAF inhibitors (vemurafenib and dabrafenib) and mitogen-activated protein kinase (MEK) inhibitors (trametinib and cobimetinib) that have shown promising results in cutaneous melanoma should also be evaluated in patients with MMM of the female genital tract $(1,23)$. A study on CIRT concomitant with immune-checkpoint inhibitors or targeted agents would be of interest to investigate the potential correlated abscopal effect. 


\section{Conclusion}

Due to its rarity, no consensus guidelines have been established for treatment of gynecological MMM, and the therapeutic approach is based on data concerning other types of gynecological cancer and cutaneous melanoma. Our preliminary data together with the Japanese data show that CIRT is a safe non-invasive option treatment for gynecological MMM, but clinical trial with a longer follow-up and larger series of patients is necessary to evaluate effectiveness and late toxicity. For this reason, we believe that a translational approach involving Gynecologic Oncologists, Medical Oncologist and Radiation Oncologists treating melanoma is desirable to improve the knowledge in the treatment of female genital tract MMM.

\section{Conflicts of Interest}

The Authors declare that there are no conflicts of interest in regard to this study.

\section{References}

1 Gadducci A, Carinelli S, Guerrieri ME and Aletti GD: Melanoma of the lower genital tract: Prognostic factors and treatment modalities. Gynecol Oncol 150: 180-189, 2018.

2 Sugiyama VE, Chan JK, Shin JY, Berek JS, Osann K and Kapp D: Vulvar melanoma: A multivariable analysis of 644 patients. Obstet Gynecol 110: 296-01, 2007.

3 Frumovitz M, Etchepareborda M, Sun CC, Soliman PT, Eifel PJ, Levenback CF and Ramirez PT: Primary malignant melanoma of the vagina. Obstet Gynecol 116: 1358-1365, 2010.

4 Kirschner AN, Kidd EA, Dewees T and Perkins SM: Treatment approach and outcomes of vaginal melanoma. Int J Gynecol Cancer 23: 1484-1489, 2013.

5 An J, Li B, Wu L, Lu H and Li N: Primary malignant amelanotic melanoma of the female genital tract: Report of two cases and review of literature. Melanoma Res 19: 267-270, 2009.

6 Seifried S, Haydu LE, Quinn MJ, Scolyer RA, Stretch JR and Thompson JF: Melanoma of the vulva and vagina: principles of staging and their relevance to management based on a clinicopathologic analysis of 85 cases. Ann Surg Oncol 22: 1959-1966, 2015.

7 Sinasac SE, Petrella TM, Rouzbahman M, Sade S, Ghazarian D and Vicus D: Melanoma of the vulva and vagina: Surgical management and outcomes based on a clinicopathologic review of 68 cases. J Obstet Gynaecol Can 18: S1701-216330582-6, 2018.

8 Leitao MM Jr., Cheng X, Hamilton AL, Siddiqui NA, JurgenliemkSchulz I, Mahner S, Åvall-Lundqvist E, Kim K and Freyer G: Gynecologic Cancer InterGroup (GCIG) consensus review for vulvovaginal melanomas Int J Gynecol Cancer 24: S117-22.7, 2014.

9 Hou JY, Baptiste C, Hombalegowda RB, Tergas AI, Feldman R, Jones NL, Chatterjee-Paer S, Bus-Kwolfski A, Wright JD and Burke WM: Vulvar and vaginal melanoma:a unique subclass of mucosal melanoma based on a comprehensive molecular metanalysis of 51 cases compared with 2253 cases of nongynecologic melanoma. Cancer 123: 1333-1344, 2013.

10 Pappa KI, Vlachos GD, Roubelakis M, Vlachos DEG, Kalafati TG, Loutradis D and Anagnou NP: Low mutational burden of eight genes involved in the MAPK/ERK, PI3K/AKT, and GNAQ/11 pathways in female genital tract primary melanomas. Biomed Res Int 15: 303791, 2015.

11 Karasawa K, Wakatsuki M, Kato S, Kiyohara H and Kamada T: Working Group for Gynecological Tumors. Clinical trial of carbon ion radiotherapy for gynecological melanoma. J Radiat Res 55: 343-350, 2014.

12 Rossi S: The National Centre for Oncological Hadrontherapy (CNAO): Status and perspectives. Phys Med 31: 333-351, 2015.

13 Giordanengo S, Garella MA, Marchetto F, Bourhaleb F, Ciocca M, Mirandola A, Monaco V, Hosseini MA, Peroni C, Sacchi R, Cirio $\mathrm{R}$ and Donetti M: The CNAO dose delivery system for modulated scanning ion beam radiotherapy. Med Phys 42: 263-275, 2015.

14 Fossati P, Molinelli S, Matsufuji N, Ciocca M, Mirandola A, Mairani A, Mizoe J, Hasegawa A, Imai R, Kamada T, Orecchia $\mathrm{R}$ and Tsujii H., Dose prescription in carbon ion radiotherapy: A planning study to compare NIRS and LEM approaches with a clinically-oriented strategy. Phys Med Biol 57: 7543-7554, 2012.

15 Molinelli S, Magro G, Mairani A, Matsufuji N, Kanematsu N, Inaniwa T, Mirandola A, Russo S, Mastella E, Hasegawa A, Tsuji H, Yamada S, Vischioni B, Vitolo V, Ferrari A, Ciocca M, Kamada T, Tsujii H, Orecchia R and Fossati P: Dose prescription in carbon ion radiotherapy: How to compare two different RBEweighted dose calculation systems. Radiother Oncol 120: 307312, 2016.

16 Krämer M and Scholz M: Treatment planning for heavy-ion radiotherapy: calculation and optimization of biologically effective dose. Phys Med Biol 45: 3319-33330, 2000.

17 Lomax A: Intensity-modulated methods for proton therapy. Phys Med Biol 44: 185-205, 1999.

18 Mirandola A, Molinelli S, Vilches Freixas G, Mairani A, Gallio E, Panizza D, Russo S, Ciocca M, Donetti M, Magro G, Giordanengo $\mathrm{S}$ and Orecchia R: Dosimetric commissioning and quality assurance of scanned ion beams at the Italian National Center for Oncological Hadrontherapy. Med Phys 42: 5287-300, 2015.

19 Molinelli S, Mairani A, Mirandola A, Vilches Freixas G, Tessonnier T, Giordanengo S, Parodi K, Ciocca M and Orecchia $\mathrm{R}$ : Dosimetric accuracy assessment of a treatment plan verification system for scanned proton beam radiotherapy: oneyear experimental results and Monte Carlo analysis of the involved uncertainties. Phys Med Biol 58: 3837-3847, 2013.

20 Pella A, Riboldi M, Tagaste B, Bianculli D, Desplanques M, Fontana G, Cerveri P, Seregni M, Fattori G, Orecchia R and Baroni G: Commissioning and quality assurance of an integrated system for patient positioning and setup verification in particle therapy. Technol Cancer Res Treat 13: 303-314, 2014.

21 National Cancer Institute: Common Terminology Criteria for Adverse Events (CTCAE), Version 4.0. Available at: http://evs.nci.nih.gov/ftp1/CTCAE/About.html. Accessed on 27 September 2018.

22 Tsujii $\mathrm{H}$ and Kamada T: A review of update clinical results of carbon ion radiotherapy. Jpn J Clin Oncol 42: 670-685, 2012.

23 Walter L and Heinzerling L: BRAF inhibitors and radiation do not act synergistically to inhibit WT and V600E BRAF human melanoma. Anticancer Res 38: 1335-1341, 2018.

Received December 14, 2018

Revised December 28, 2018

Accepted January 7, 2019 